VoL. $62(2000) \quad[149-154]$

\title{
ALMOST ALL EXTRASPECIAL $p$-GROUPS ARE SWAN GROUPS
}

\author{
David John Green and Pham Anh Minh
}

Let $P$ be an extraspecial $p$-group which is neither dihedral of order 8 , nor of odd order $p^{\mathbf{3}}$ and exponent $p$. Let $G$ be a finite group having $P$ as a Sylow $p$-subgroup. Then the mod- $p$ cohomology ring of $G$ coincides with that of the normaliser $N_{G}(P)$.

\section{INTRODUCTION}

Let $P$ be a finite $p$-group. Martino and Priddy call $P$ a Swan group [5] if for every finite group $G$ with Sylow $p$-subgroup $P$, the mod-p cohomology ring $H^{*}(G)$ coincides with $H^{*}\left(N_{G}(P)\right) \cong H^{*}(P)^{N_{G}(P)}$. In particular, if there are no so-called transfer summands in the stable decomposition of the classifying space $B P$, then $P$ is a Swan group.

We prove in Theorem 3.3 that all extraspecial p-groups are Swan groups, apart from the well-known exceptions $2_{+}^{1+2}=D_{8}$ and (for $p$ odd) $p_{+}^{1+2}=E$. The cases where $P$ is the metacyclic group $p_{-}^{1+2}=M\left(p^{3}\right)$ with $p$ odd, and where $P$ is $2_{-}^{1+2 n}=Q_{8} * D_{8} * \cdots * D_{8}$, were proved in [5]; the former case being due to G. Glauberman. Earlier, the $p=2$ case of the theorem was published in [7], but with an incorrect proof: see Remark 3.6. In Corollary 3.5 we generalise another result of Martino and Priddy, exhibiting three infinite families of Swan groups whose classifying spaces do have transfer summands in their stable decompositions.

Throughout this paper we denote the mod- $p$ cohomology ring $H^{*}\left(G, \mathbb{F}_{p}\right)$ by $H^{*}(G)$. A suitable reference on group cohomology is Evens' book [2].

\section{EXTRASPECIAL $p$-GROUPS}

Recall that a $p$-group $P$ is called extraspecial if its centre $Z(P)$, its derived subgroup $P^{\prime}$ and its Frattini subgroup $\Phi(P)$ all coincide, and are cyclic of order $p$. So if $P$ is extraspecial there is a central extension

$$
1 \rightarrow \mathbb{F}_{p} \longrightarrow P \stackrel{\psi}{\longrightarrow} V \rightarrow 1
$$

with $V$ an elementary Abelian $p$-group. Hence there is a nondegenerate alternate bilinear form $f$ on $V$ defined by $f(\psi(g), \psi(h))=[g, h]$ for all $g, h \in P$.

Received 8th November, 1999

Copyright Clearance Centre, Inc. Serial-fee code: 0004-9727/00 \$A2.00+0.00. 
Moreover, for $p$ odd there is a linear form $\lambda$ on $V$ defined by $\lambda(\psi g)=g^{p}$; and for $p=2$ there is a quadratic form $Q$ on $V$ defined by $Q(\psi g)=g^{2}$ with associated bilinear form $f$. Conversely, such a pair $(\lambda, f)$ determines an extraspecial $p$-group when $p$ is odd, and such a $Q$ determines an extraspecial 2-group.

See [4, Chapter 6] for a reference on alternate bilinear forms, and $[1,1.16]$ for a reference on quadratic forms in characteristic 2 . Nondegeneracy means that $V$ has even dimension, say $2 n$. Up to change of basis for $V$ there are two possibilities for $Q$ when $p=2$, and exactly one choice of $f$ for odd $p$. For $p$ odd there are two possibilities for the pair $(\lambda, f)$ : either $\lambda$ is identically zero, or it is not. Note that in the case of nonzero $\lambda$, Witt's extension theorem does not hold for the pair $(\lambda, f)$, as the restriction of $f$ to $\operatorname{ker}(\lambda)$ does have a kernel.

So there are four types of extraspecial $p$-groups. In each case $P$ is generated by $A_{1}, \ldots, A_{n}, B_{1}, \ldots, B_{n}, C$, with $C$ central of order $p,\left[A_{i}, A_{j}\right]=\left[B_{i}, B_{j}\right]=1$ and $\left[A_{i}, B_{j}\right]=C^{\delta_{i j}}$. Moreover $A_{i}^{p}=B_{i}^{p}=1$ for $2 \leqslant i \leqslant n$. The four cases are:

- $2_{+}^{1+2 n}=D_{8} * \cdots * D_{8}$ : here $A_{1}^{2}=B_{1}^{2}=1$.

- $2_{-}^{1+2 n}=Q_{8} * D_{8} * \cdots * D_{8}$ : here $A_{1}^{2}=B_{1}^{2}=C$.

- $p_{+}^{1+2 n}=E * \cdots * E$ has odd exponent $p$ : here $A_{1}^{p}=B_{1}^{p}=1$.

- $p_{-}^{1+2 n}=M\left(p^{3}\right) * E * \cdots * E$ has odd exponent $p^{2}$ : here $A_{1}^{p}=C$ and $B_{1}^{p}=1$.

The characteristic subgroup $\Omega_{1}(P)$ of $P$ is the subgroup generated by all order $p$ elements. Denote $Z\left(\Omega_{1}(P)\right)$ by $Y$. If $P$ has odd exponent $p^{2}$ then $Y=\left\langle B_{1}, C\right\rangle$ is rank two elementary Abelian; in all other cases, $Y$ equals $Z=Z(P)$.

The following result could be called Witt's theorem for extraspecial p-groups.

PRoposition 1.1. Let $P$ be an extraspecial $p$-group. Suppose that $H, K$ are subgroups of $P$ containing $Z$, and that $\phi: H \rightarrow K$ is a group isomorphism inducing the identity map on $Z$. If $P$ has odd exponent $p^{2}$, assume further that $H \cap Y=K \cap Y$ and that $\phi$ induces the identity map on $(H \cap Y) / Z$. Then $\phi$ extends to an automorphism of $P$.

Lemma 1.2. In Proposition 1.1, suppose that $P$ has odd exponent $p^{2}$ and that $H \cap Y$ is $Z$ rather than $Y$. Then $\phi$ extends to an isomorphism from $\langle H, Y\rangle$ to $\langle K, Y\rangle$ which itself satisfies the conditions of Proposition 1.1.

Proof: Since $\phi(C)=C$ it follows that $h^{-1} \phi(h)$ lies in $\operatorname{ker}(\lambda)$ for every $h \in H$. Hence $\phi\left(\left[h, B_{1}\right]\right)=\left[\phi(h), B_{1}\right]$. So we may set $\phi\left(B_{1}\right)=B_{1}$.

Proof of Proposition 1.1: Denote by $U, W$ the images in $V$ of $H, K$ respectively. Since $\phi$ is the identity on $Z$ there is an $F_{p}$-vector space isomorphism $\rho: U \rightarrow W$ induced by $\phi$ which respects the alternate bilinear form $f$ on $V$.

If $p$ is 2 then $\rho$ respects the quadratic form $Q$. Since Witt's extension theorem holds for $Q$ (see $[1$, p. 36]), we may extend $\rho$ to a $Q$-orthogonal transformation $\rho$ of $V$. Using the standard generators for $P$ we may lift $\rho$ to an automorphism $\phi^{\prime}$ of $P$. If $h \in H$ then 
$\phi^{\prime}(h)=\phi(h) C^{r}$ for some $r \in \mathbb{Z} / p$. Since $P$ has enough inner automorphisms, we may assume that $\phi^{\prime}$ extends $\phi$.

To be more precise: pick $h_{1}, \ldots, h_{m} \in H$ whose images under $\psi: P \rightarrow V$ constitute a basis for $U$. Since the alternate bilinear form $f$ on $V$ is nondegenerate we can pick $g_{1}, \ldots, g_{m} \in P$ such that $f\left(\psi\left(g_{i}\right), \psi\left(\phi h_{j}\right)\right)=\delta_{i j}$. Hence conjugation by $g_{i}$ fixes $\phi\left(h_{j}\right)$ for $j \neq i$ and sends $\phi\left(h_{i}\right)$ to $\phi\left(h_{i}\right) C$. So we can correct $\phi^{\prime}$ by an inner automorphism of $P$ to ensure that $\phi^{\prime}=\phi$ on $H$.

Now suppose that $p$ is odd. As Witt's extension theorem holds for $f$ (see $[4,6.9]$ ), we may extend $\rho$ to a transformation of $V$ that respects $f$. But then $\rho$ respects $\lambda$ too: this is trivial in the exponent $p$ case, as $\lambda$ is then zero. In the exponent $p^{2}$ case, we may assume by Lemma 1.2 that $H, K$ contain $Y$ and that $\rho$ fixes $\psi\left(B_{1}\right)$. So $\rho$ respects $\lambda$ by Lemma 1.3 below. As in the $p=2$ case we can now lift $\rho$ to an automorphism of $P$ which extends $\phi$.

LEMma 1.3. Suppose $P$ is an extraspecial p-group with odd exponent $p^{2}$. Taking $C$ as basis for $Z \cong \mathbb{F}_{p}$, we may assume that $\lambda\left(\psi A_{1}\right)=1$. Then for any $v \in V$ we have $\lambda(v)=f\left(v, \psi\left(B_{1}\right)\right)$.

PROOF: Each element $g$ of $P$ has canonical form $B_{1}^{s_{1}} \cdots B_{n}^{s_{n}} \cdot A_{1}^{r_{1}} \cdots A_{n}^{r_{n}} \cdot C^{t}$. Then $g^{p}=C^{r_{1}}=\left[g, B_{1}\right]$.

\section{LOCAL SUBGROUP STRUCTURE}

Throughout this section $G$ is a finite group with extraspecial Sylow $p$-subgroup $P$.

Lemma 2.1. Suppose that $P$ is not one of $D_{8}, E, M\left(p^{3}\right)$. Then for any order $p$ element $g$ of $P$, the centraliser of $g$ in $P$ has the same Frattini subgroup as $P$ itself.

PRoof: If $n \geqslant 2$ then $C_{P}(g)$ is not Abelian. In $Q_{8}$, all order $p$ elements are central. The other three groups really are exceptions: take $g=B_{1}$.

LEMMA 2.2. Suppose that the centraliser of each exponent $p$ element of $P$ has $Z$ as its Frattini subgroup. Then $Z^{g}=Z$ for every $g \in G$ such that $P \cap P^{g}$ contains $Z$. Moreover, such $g$ factorise as $g=g_{1} g_{2}$ with $g_{1} \in N_{G}(P)$ and $g_{2} \in C_{G}(Z)$.

Proof: Observe that $P$ is a Sylow $p$-subgroup of $C_{G}(Z)$, and so all Sylow $p$ subgroups of $C_{G}(Z)$ have Frattini subgroup $Z$. Now set $R$ equal to $P^{g} \cap C_{G}(Z)$. By assumption the Frattini subgroup of $R$ is that of $P^{g}$, namely $Z^{g}$. But $R$ is contained in a Sylow $p$-subgroup of $C_{G}(Z)$. We conclude that $Z^{g}=Z$.

Therefore $P^{g}$ is itself a Sylow p-subgroup of $C_{G}(Z)$, and so $P^{g}=P^{h}$ for some $h \in C_{G}(Z)$. Take $g_{1}=g h^{-1}$ and $g_{2}=h$.

Lemma 2.3. Suppose that $P$ has odd exponent $p^{2}$. Then $Y^{g}=Y$ for every $g \in C_{G}(Z)$ such that $Y \leqslant P \cap P^{g}$. Moreover, such $g$ factorise as $g_{1} g_{2}$, where $g_{1}$ lies in $C_{G}(Z) \cap N_{G}(P)$ and $g_{2} \in C_{G}(Z) \cap N_{G}(Y)$ acts trivially on $Y / Z$. 
Proof: Set $D_{1}$ equal to $C_{G}(Y)$, which contains $\Omega_{1}(P)$. Since the centre of $P$ is cyclic and $\Omega_{1}(P)$ is maximal in $P$, it follows that $\Omega_{1}(P)$ is a Sylow $p$-subgroup of $D_{1}$. Now let $R$ be $P^{g} \cap D_{1}$, the centraliser of $Y$ in $P^{g}$. Since $\Omega_{1}(P)$ has exponent $p$, so does $R$. As $g$ centralises $Z$, we deduce that $R$ is a maximal subgroup of $P^{g}$. But the only exponent $p$ maximal subgroup of $P^{g}$ is $\Omega_{1}\left(P^{g}\right)$, which has centre $Y^{g}$. So $g$ normalises $Y$.

Now set $D_{2}=\left\{h \in C_{G}(Z) \cap N_{G}(Y) \mid g\right.$ acts trivially on $\left.Y / Z\right\}$. Then $P$ is a Sylow $p$-subgroup of $D_{2}$, and $P^{g}$ is too since $g$ lies in $C_{G}(Z) \cap N_{G}(Y)$. So $P^{g}=P^{h}$ for some $h \in D_{2}$. Take $g_{1}=g h^{-1}$ and $g_{2}=h$.

\section{STABILITY CONDITIONS}

The following elementary reformulation of the usual stability condition is not new, but does not appear to be widely known.

Lemma 3.1. Let $P$ be a Sylow p-subgroup of a finite group $G$. The cohomology class $x \in H^{*}\left(P, \mathbb{F}_{p}\right)$ lies in $\operatorname{Im} \operatorname{Res}_{P}^{G}$ if and only if $x$ is an $N_{G}(P)$-invariant and

$$
\operatorname{Cor}_{P \cap P^{g}}^{P} \operatorname{Res}_{P \cap P^{g}}^{P^{g}} g^{*}(x)=0 \quad \text { for all } g \in G-N_{G}(P) \text {. }
$$

Proof: If $x$ comes from $H^{*}(G)$, then it certainly satisfies both conditions. Conversely, observe that the conditions combined with the Mackey formula mean that $\operatorname{Res}_{P}^{G} \operatorname{Cor}_{P}^{G}(x)=\left|N_{G}(P): P\right| x$.

Let $P$ be a finite $p$-group. The ring of universally stable elements $I(P)$ was defined in [3] as the subring of $H^{*}\left(P, \mathbb{F}_{p}\right)$ given by

$$
I(P)=\bigcap_{G} \operatorname{Im} \operatorname{Res}_{P}^{G},
$$

where $G$ ranges over all finite groups with Sylow $p$-subgroup $P$. The following observation appears in [7]. Recall that $O^{p}(G)$ is the subgroup generated by all $p^{\prime}$-elements of $G$.

LEMMA 3.2. $I(P) \subseteq H^{*}(P)^{\text {OP(Out }(P))}$.

Proof: Pick any outer automorphism of order prime to $p$, and lift it to an automorphism $\alpha$ of the same order. Let $G$ be the semidirect product $P \rtimes\langle\alpha\rangle$.

It is immediate that if $P$ is a Swan group then equality holds in Lemma 3.2. The main result of this paper is:

Theorem 3.3. All extraspecial p-groups $P$ apart from $D_{8}$ and $E$ are $S$ wan groups. If $P$ is $D_{8}$ or $E$, then the universally stable elements for $P$ are strictly contained in $H^{*}(P)^{\text {OP(Out }(P))}$.

Proof: See [5] for a proof that $M\left(p^{3}\right)$ is a Swan group. The groups $D_{8}$ and $E$ are treated in Lemma 3.4 below. So we may assume that $P$ satisfies the hypotheses of Lemma 2.1 and hence those of Lemma 2.2. 
Let $G$ be a finite group with extraspecial Sylow p-subgroup $P$, and let $x \in H^{*}(P)$ be an $N_{G}(P)$-invariant. We show that the conditions of Lemma 3.1 are satisfied. Let $g$ be an element of $G-N_{G}(P)$. If $P^{g} \cap P$ is elementary Abelian but not maximal in $P$, then corestriction from $P^{g} \cap P$ to $P$ is zero: for corestriction from any group $H$ to $H \times C_{p}$ is zero.

We may therefore assume that $P^{g}, P$ both contain $Z=\Phi(P)$. Write $H$ for $P \cap P^{g}$ and $L$ for ${ }^{g} P \cap P$. By Lemma 2.2 we deduce that $g$ normalises $Z$. Moreover, since $x$ is invariant under $N_{G}(P)$, we may in fact assume that $g$ stabilises $Z$. If $P$ has odd exponent $p^{2}$ we deduce further by Lemma 2.3 either that $g$ normalises $Y$ and can be taken to act trivially on $Y / Z$, or that $H \cap Y=L \cap Y=Z$.

So we can now apply Proposition 1.1 and deduce that conjugation $c_{g}: H \rightarrow L$ extends to an automorphism $\phi$ of $P$. Then $\operatorname{Res}_{H}^{P g} g^{*}=\operatorname{Res}_{H}^{P} \phi^{*}$, which means that $\operatorname{Cor}_{H}^{P} \operatorname{Res}_{H}^{P g} g^{*}=0$ as $H$ is a proper subgroup of $P$.

Lemma 3.4. If $P$ is $D_{8}$ or $E$, then the inclusion in Lemma 3.2 is strict.

Proof: Let $G$ be $G L_{3}\left(\mathbb{F}_{p}\right)$. Then the upper triangular matrices with ones on the diagonal form a Sylow $p$-subgroup isomorphic to $P$. We may take $B_{1}=\left(\begin{array}{lll}1 & 0 & 0 \\ 0 & 1 & 1 \\ 0 & 0 & 1\end{array}\right)$ and
$C=\left(\begin{array}{lll}1 & 0 & 1 \\ 0 & 1 & 0 \\ 0 & 0 & 1\end{array}\right)$. Pick $g=\left(\begin{array}{lll}0 & 1 & 0 \\ 1 & 0 & 0 \\ 0 & 0 & 1\end{array}\right)$. Then $F=P \cap P^{g}$ equals $\left\langle B_{1}, C\right\rangle$, a maximal elementary Abelian subgroup. Let $\beta, \gamma$ be the dual basis for $F^{*}$.

Taking the first Chern class is an $\mathbb{F}_{p}$-linear monomorphism from $F^{*}$ to $H^{2}(F)$. Let $\rho$ be an ordinary representation of $P$ with character $\chi$, the sum of all $p^{2}$ linear characters. These linear characters restrict to $F$ as scalar multiples of $\beta$, each scalar multiple being the image of $p$ characters. So by the Whitney sum formula, the total Chern class $c(\rho)$ restricts to $F$ as follows:

$$
\operatorname{Res}_{F}^{P} c(\rho)=\prod_{\mu \in \mathbb{P}_{p}}(1+\mu \beta)^{p} .
$$

This equals $1-\beta^{p(p-1)}$. Set $\eta$ equal to $c_{p(p-1)}(\rho)$ in $H^{2 p(p-1)}(P)$. Then $\eta$ lies in $H^{*}(P)^{O^{P}(O u t(P))}$, since $\chi$ is an invariant of $\operatorname{Aut}(P)$. But $\operatorname{Res}_{F}^{P}(\eta)=-\beta^{p(p-1)}$ is distinct from $g^{*} \operatorname{Res}_{F}^{P}(\eta)=-\gamma^{p(p-1)}$, so $\eta$ is not stable.

Suppose that $P$ is a $p$-group whose classifying space does not have any transfer summands in its stable splitting. Then $P$ is a Swan group by [5, Theorem 3.5]. Martino and Priddy give one counterexample to the converse: $M\left(p^{3}\right)$.

Corollary 3.5. Let $P$ be $2_{+}^{1+2 n}$ with $n \geqslant 2$; or $p_{+}^{1+2 n}$ with $p$ odd and $n \geqslant 2$; or $p_{-}^{1+2 n}$ with $p$ odd and $n \geqslant 1$. Then although $P$ is a Swan group, the stable splitting of $B P$ involves a transfer summand. 
ProOF: $F=\left\langle B_{1}, \ldots, B_{n}, C\right\rangle$ is a self-centralising, maximal elementary Abelian subgroup. By [8, Theorem 0.1], the Steinberg summand $L(n+1)$ of $B F$ is a transfer summand of $B P$.

REMARK 3.6. Ogawa's proof of Theorem 3.3 hinges around the following claim: if $M$ is a maximal subgroup of an extraspecial 2-group $P \neq D_{8}$, then $P$ acts trivially on $H^{*}(M)$. As observed in [6], the proof of this claim in [7] uses inflation incorrectly. We shall now see that the claim is false for $2_{+}^{1+2 n}$.

Let $P$ be $D_{8} * \cdots * D_{8}$, or $p_{+}^{1+2 n}$ if $p$ is odd. Let $M$ be $C_{P}\left(B_{1}\right)$, an index $p$ subgroup of $P$. Then $F=\left\langle B_{1}, \ldots, B_{n}, C\right\rangle$ is maximal elementary Abelian in $M$ and in $P$. Let $\beta_{1}, \ldots, \beta_{n}, \gamma$ be the dual basis of $F^{*}$, which we embed in $H^{2}(F)$ by taking the first Chern class. Then $S\left(F^{*}\right) \subseteq H^{*}(F)$. Define $\zeta \in H^{2 p^{n-1}}(M)$ to be the Evens norm $N_{F}^{M}(\gamma)$, and set $g=A_{1}$. Let $U$ be the subspace of $F^{*}$ spanned by $\beta_{2}, \ldots, \beta_{n}$. By standard properties of the Evens norm map (see [2, Chapter 6]), we have:

$$
\operatorname{Res}_{F}^{M}(\eta)=\prod_{u \in U}(\gamma+u) \quad \text { and so } \quad g^{*} \operatorname{Res}_{F}^{M}(\eta)=\prod_{u \in U}\left(\gamma+\beta_{1}+u\right)
$$

These restrict to $\left\langle B_{1}, C\right\rangle$ as $\gamma^{p^{n-1}}$ and $\left(\gamma+\beta_{1}\right)^{p^{n-1}}$ respectively. So $\operatorname{Res}_{F}^{M}\left(g^{*} \eta\right)$ differs from $\operatorname{Res}_{F}^{M}(\eta)$, which means that $\eta$ is not invariant under $A_{1}$.

\section{REFERENCES}

[1] J.A. Dieudonné, La géométrie des groupes classiques, Ergebnisse der Mathematik und ihrer Grenzgebiete, Band 5, (third edition) (Springer-Verlag, Berlin, Heidelberg, New York, 1971).

[2] L. Evens, The cohomology of groups, Oxford Mathematical Monographs (Oxford University Press, Oxford, 1991).

[3] L. Evens and S. Priddy, 'The ring of universally stable elements', Quart. J. Math. Oxford Ser. (2) 40 (1989), 399-407.

[4] N. Jacobson, Basic algebra. I, (second edition) (W.H. Freeman and Company, New York, 1985).

[5] J. Martino and S. Priddy, 'On the cohomology and homotopy of Swan groups', Math. Z. 225 (1997), 277-288.

[6] P.A. Minh, 'Proper singularities of extraspecial p-groups for the mod-p cohomology functor', Comm. Algebra 25 (1997), 965-971.

[7] Y. Ogawa, 'On the subring of universally stable elements in a mod-2 cohomology ring', Tokyo J. Math. 15 (1992), 91-97.

[8] S.B. Priddy, 'On characterizing summands in the classifying space of a group. I', Amer. J. Math. 112 (1990), 737-748.

Department of Mathematics

University of Wuppertal

D-42097 Wuppertal

Germany

e-mail: green@math.uni-wuppertal.de
Department of Mathematics

University of Hue

27 Nguyen Hue

Hue

Vietnam 